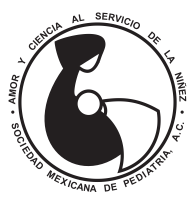

\title{
Nuevos y viejos retos quirúrgicos asociados a la pandemia COVID-19
}

\author{
José Francisco González-Zamora ${ }^{\ddagger} * *$ \\ ${ }^{\ddagger}$ Editor Asociado, Revista Mexicana de Pediatría. Cirujano Pediatra, Investigador en Ciencias Médicas, Instituto Nacional de \\ Pediatría, Ciudad de México.
}

En un mundo donde la efectividad y seguridad de la actividad quirúrgica alcanzan grandes beneficios, la inequidad entre las poblaciones marca una diferencia dolorosa. Con la finalidad de abordar esta brecha, en mayo de 2015 la Organización Mundial de la Salud (OMS) adopta la resolución WHA68.15, que reconoce a la atención quirúrgica como un componente indispensable de la cobertura universal de salud, y además compromete a los países miembros para el desarrollo de programas quirúrgicos integrales, que deberán ser incluidos en los planes nacionales de salud de cada gobierno. Aunado a esto, se reconoce que el requerimiento de atención quirúrgica aumenta con el tiempo. ${ }^{1}$

En la actualidad, se estima que se requiere de un procedimiento quirúrgico por cada 21 sujetos vivos, y posiblemente sea mayor. ${ }^{2}$ Lograr la cobertura con la calidad planeada representa un reto a los sistemas de salud del mundo, principalmente de países con una baja inversión en salud como México.

En este difícil escenario, el 11 de marzo de 2020 la OMS declara a la enfermedad COVID-19 (Coronavirus Disease 2019) como pandemia, con implicaciones socioeconómicas globales de magnitud por definir y, por supuesto, con impacto negativo en la atención quirúrgica a nivel mundial. Esta pandemia llega a México en un momento en donde el acceso a servicios quirúrgicos seguros aún es limitado. Los problemas en la actividad quirúrgica que se visualizan en nues-

\footnotetext{
*Correspondencia: JFGZ, jf.gonzalezzamora@gmail.com Conflicto de intereses: El autor declara que no tiene. Citar como: González-Zamora JF. Nuevos y viejos retos quirúrgicos asociados a la pandemia COVID-19. Rev Mex Pediatr. 2020; 87(3):83-85. doi: $10.35366 / 94836$

[New and old surgical challenges related to the COVID-19 pandemic]
}

tro país no sólo se asocian a la implementación de medidas globales dirigidas al control de riesgos por infección de SARS-CoV-2 en personal para la salud y pacientes, sino también deben incluir el rezago en infraestructura, recursos y programas de atención quirúrgica que ya teníamos. Estos problemas se especifican a continuación con una visión dirigida al campo de la Pediatría.

Existe poca evidencia sobre la actividad quirúrgica infantil en México, quizá asociada a un subregistro nacional. En los últimos 10 años, el reporte global sobre la actividad quirúrgica realizada por la Secretaría de Salud muestra para el 2019 una disminución de 1.6 a 1.3 millones de procedimientos quirúrgicos, en donde la cirugía infantil representó entre el $14-15 \%,{ }^{3}$ con un descenso en la tendencia nacional de procedimientos quirúrgicos desde el 2017.

Trece días después de la declaración de pandemia por COVID-19, el American College of Surgeon (ACS) emite lineamientos para el tamizaje de cuidados quirúrgicos electivos en cirugía pediátrica, los cuales fueron adoptados por muchos países de América. El objetivo fue proporcionar atención quirúrgica oportuna a niños con problemas quirúrgicos pediátricos de emergencia y urgencia, al tiempo que se optimizan los recursos de atención hospitalaria para los pacientes, pero teniendo en cuenta la preservación de la salud de los cuidadores. Así, ACS determina que la cirugía electiva debería ser retrasada.

Inicialmente, el diferimiento de las cirugías fue adoptada en el mundo con diferentes resultados, de acuerdo con la incidencia de la enfermedad, población y sistemas de salud. Sin embargo, ¿qué pasaría si la pandemia tardara meses en ser controlada? ¿Cuánto tiempo más podremos mantener la actividad quirúrgica electiva, en espera? 
A nivel mundial, conforme ha permanecido la contingencia sanitaria, muchos gobiernos y organismos profesionales están pasando de una posición de restricción a la reapertura de cirugía electiva. Ante la reapertura, distintas estrategias se han propuesto para tratar de disminuir los riesgos: estratificar los procedimientos de acuerdo con los recursos disponibles, establecer una programación quirúrgica escalonada que incluya los procedimientos electivos y se ajuste a la incidencia local de COVID-19, impulsar la implementación de unidades quirúrgicas ambulatorias independientes "libres" de COVID-19, formación y fortalecimiento de equipos quirúrgicos colaborativos entre los distintos hospitalarios y comunidades. ${ }^{4}$ Incluso se plantea una propuesta para priorizar el inicio de la cirugía electiva con base en un sistema de valores estandarizado (MeNTS). ${ }^{5}$ En algo que todos los grupos han coincidido, es en la necesidad de crear guías institucionales basadas en experiencia quirúrgica local, recursos hospitalarios disponibles y las condiciones geopolíticas de cada población; ${ }^{6}$ en nuestro país recién iniciamos en su desarrollo.

La evidencia científica sobre lo que está sucediendo con la actividad quirúrgica hospitalaria aún está en desarrollo, y son pocos los reportes en el campo pediátrico. La COVIDSurg Collaborative reportó los resultados de un estudio de cohorte internacional donde se evaluaron los primeros 30 días postoperatorios en 1,128 adultos con COVID-19, que fueron sometidos a una amplia gama de procedimientos quirúrgicos. ${ }^{7} \mathrm{El}$ resultado primario fue la mortalidad postoperatoria en el $23.8 \%$ de la población, asociado principalmente con infecciones respiratorias graves. Llamó la atención que el $71 \%$ de estas infecciones fue diagnosticado en el periodo postoperatorio, lo que supone que el contagio fue posterior a la intervención y durante el pico de mayor incidencia. Estos resultados impactaron en la actividad quirúrgica mundial, porque la tasa de malos resultados supera por mucho los observados de manera habitual. El proceso inflamatorio severo y el estado protrombótico que se asocia con COVID-19 parecen incrementarse por la cirugía y la inmovilización postquirúrgica habitual, por lo que se han iniciado múltiples proyectos en el mundo, para tratar de disminuir la morbilidad.

La atención quirúrgica infantil representa un grupo pequeño de la atención quirúrgica global, de la que se diferencia en dos puntos sustanciales: 1) los niños tienen una tasa de morbilidad y mortalidad preoperatoria más baja que los adultos, y 2) la comorbilidad preoperatoria también es mucho menor. Estos puntos hacen una diferencia importante al analizar la información generada en adultos y extrapolarla a niños.

Recientemente, en un reporte de un estudio multicéntrico de hospitales pediátricos en EUA que evaluó 1,295 niños sin el antecedente clínico de COVID-19, se realizó la detección de virus SARS-CoV-2 por RT-PCR, previo a ser sometidos a una intervención quirúrgica. ${ }^{8}$ El resultado primario determinó que la incidencia de COVID-19 ocurrió en menos del 1\%, y estableció diferencias de acuerdo con cada hospital incluido. La mitad de los pacientes no presentaron síntomas al momento de la revisión clínica preoperatoria y aquéllos que los presentaron pudieron confundirse con la enfermedad que condicionó el procedimiento. De esta forma, la baja sensibilidad del cribado clínico, obliga a la realización de pruebas diagnósticas preoperatorias en todos los niños que serán sometidos a un procedimiento quirúrgico, para la protección tanto del paciente como del personal encargado de su atención perioperatoria. Desafortunadamente, es una medida que no se realiza de rutina en los hospitales pediátricos del mundo; en nuestro país no tenemos información sobre el uso o impacto de la aplicación de rutina de esta prueba. Aún están en proceso líneas de investigación que evalúen la morbilidad y mortalidad postoperatoria en población infantil.

En resumen, la cirugía en todo el mundo se ha visto afectada por esta pandemia, los retos previos se magnificaron y aparecieron otros. La forma en la que practicamos la atención quirúrgica, la enseñamos y la aprendemos está cambiando. Lejos de amedrentarnos, es una oportunidad de cambio, de plantearnos nuevos procesos y buscar el trabajo colaborativo. Esta pandemia puso de manifiesto en nuestro país los viejos retos para el acceso universal a una atención quirúrgica segura, y plantea nuevos retos por definir. Como bien lo describe la Dra. Barrientos-Gutiérrez en relación con esta pandemia en México, ${ }^{9}$ nuestra función primordial es proteger la salud de la sociedad mexicana con ideas que partan de un análisis crítico, científico y creativo, siempre con la colaboración de todos los involucrados en la toma de decisiones en salud.

\section{REFERENCIAS}

1. Price R, Makasa E, Hollands M. World Health Assembly Resolution WHA68.15: "Strengthening emergency and essential surgical care and anesthesia as a component of universal health coverage"Addressing the public health gaps arising from lack of safe, affordable and accessible surgical and anesthetic services. World J Surg. 2015; 39(9): 2115-2125. doi: 10.1007/s00268-015-3153-y

2. Weiser TG, Haynes AB, Molina G et al. Size and distribution of the global volume of surgery in 2012. Bull World Health Organ. 2016; 94(3): 201-209F. doi: 10.2471/BLT.15.159293. 
3. Dirección General de Información en Salud. Egresos hospitalarios. Cubos dinámicos. México: Secretaría de Salud; 20 de julio 2020. Disponible en: http://www.dgis.salud.gob.mx/contenidos/ basesdedatos/bdc_egresoshosp_gobmx.html

4. Al-Jabir A, Kerwan A, Nicola M et al. Impact of the Coronavirus (COVID-19) pandemic on surgical practice - Part 1. Int J Surg. 2020; 79: 168-179. doi: 10.1016/j.ijsu.2020.05.022.

5. Slidell MB, Kandel JJ, Prachand V et al. Pediatric modification of the medically necessary, time-sensitive scoring system for operating room procedure prioritization during the COVID-19 pandemic. J Am Coll Surg. 2020;231(2):205-215. doi: 10.1016/j.jamcollsurg.2020.05.015.

6. Diaz A, Sarac BA, Schoenbrunner AR, Janis JE, Pawlik TM. Elective surgery in the time of COVID-19. Am J Surg. 2020; 219(6): 900-902. doi: 10.1016/j.amjsurg.2020.04.014.
7. COVIDSurg Collaborative. Mortality and pulmonary complications in patients undergoing surgery with perioperative SARS-CoV-2 infection: an international cohort study [published correction appears in Lancet. 2020 Jun 9;]. Lancet. 2020; 396(10243): 27-38. doi: 10.1016/S0140-6736(20)31182-X.

8. Lin EE, Blumberg TJ, Adler AC et al. Incidence of COVID-19 in pediatric surgical patients among 3 US children's hospitals [published online ahead of print, 2020 Jun 4]. JAMA Surg. 2020; e202588. doi: 10.1001/jamasurg.2020.2588.

9. Barrientos-Gutiérrez T, Alpuche-Aranda C, Lazcano-Ponce E, Pérez-Ferrer C, Rivera-Dommarco J. La salud pública en la primera ola: una agenda para la cooperación ante COVID-19. Salud Publica Mex. 2020; 10.21149/11606. doi: $10.21149 / 11606$. 